\title{
Banana production in Brazil and Jamaica
}

This time we focus on two papers of great historical interest from vol. 12 (8) and (9) Fruits issues that are monographs on banana production in Brazil and Jamaica. They were published in fact around the time of the arrival of Panama disease in both countries. In Brazil, this resulted in the almost complete extinction of the Maça banana, and, in Jamaica, in the replacement of the Gros Michel, on a purely provisional basis it was thought at the time, by Lacatan, a Cavendish type.

Some interesting insights are given on Sigatoka, which had appeared 20 years beforehand in Jamaica, but had been present for only 5 years in Brazilian production zones.

Here are some extracts from the article by Julio Di Paravicini Torres, "The Banana in Brazil":

"The State of São Paulo is the largest banana producer in Brazil [...]. The cultivation [...] is ancient, constituting the principal source of income of the "paulista" littoral.

The "Maça" banana (or Apple banana) is much appreciated in Brazil [...]. Recently its cultivation has declined strongly due to its susceptibility to "Panama disease". The municipality of Piracicaba [...], considered as the largest producer in Brazil of this variety, had more than 1,000,000 plants in 1930 that were completely decimated by this disease. At that time, some plant pathologists thought that it was a new disease, which they named "mal de Piracicaba" [...].

The "mal de Sigatoka" was notified in Amazonia in 1942 and was identified in São Paulo in 1952 [...].

For the South American markets (Argentina, Uruguay), the bunches are fastened in the ventilated bolds or on the bridge of the boats for trips lasting two and a half to three days. For the European countries for which the voyage is 15 to 17 days (England, Germany and other countries), the bunches must be packed and loaded into refrigerated bolds.

In Santos, there are factories for banana flour, banana paste and other foodstuffs."

And some from the article by Pierre Subra, "Characteristics of banana production in Jamaica":

"The essential factor for progress is varietal change following the progressive destruction of Gros-Michel by Panama Disease. This disappearance is increasingly complete and it seems that the areas still barely touched two years ago [...] very soon will be [...]. It is thus possible to say that the Jamaican variety is Lacatan [...]. Although this variety has always been regarded as provisional, it is likely that producers and consumers alike will end up adopting it.

Sigatoka disease, which appeared in Jamaica around 1936, has been the subject of many investigations, particularly by Dr. Leach, currently the Head of Research at the Banana Board [...]. Banana breeding work has been carried out in Trinidad since well before the last war [...]. Currently there are two complementary phases:

- Imperial College, Trinidad, with Dr. Simmonds, is studying, selecting and multiplying the male parents,

- the Bodles Breeding Station, Jamaica, then receives the selected male parents, carries out pollinations and studies the hybrids obtained." 


\section{Lu dans Fruits, il y a 50 ans...}

\section{Production de bananes au Brésil et à la Jamaïque}

Les deux articles des numéros 8 et 9 du volume 12 de Fruits qui ont retenu notre attention sont des monographies de la production de banane au Brésil et à la Jamaïque qui revêtent un grand intérêt historique Ils ont en effet été publiés au moment de l'arrivée de la maladie de Panama dans ces zones, se traduisant au Brésil par la quasi extinction du bananier Maça et, à la Jamaïque, par le remplacement, à titre provisoire pensait-on à l'époque, du bananier Gros Michel par un type Cavendish, le Lacatan.

Des indications intéressantes sont données sur la maladie de Sigatoka, apparue 20 ans auparavant à la Jamaïque, mais présente seulement depuis 5 ans dans la zone de culture brésilienne.

Voici quelques extraits de l'article de Julio Di Paravicini Torres " la Banane au Brésil " :

"L'Etat de São Paulo est le plus gros producteur de bananes du Brésil [...]. La culture [...] est ancienne, constituant la principale source de revenu du littoral pauliste.

La banane "Maça" (banane Pomme) est très appréciée des Brésiliens [...]. Dernièrement elle a été peu cultivée du fait de la sensibilité à la " maladie de Panama ". Le municipe de Piracicaba, [...] considéré comme le plus gros producteur du Brésil de cette variété, possédait en 1930 plus de 1000000 de pieds qui ont été complètement décimés par cette maladie. A cette époque quelques phytopathologistes pensaient qu'il s'agissait d'une nouvelle maladie qu'ils nommèrent " mal de Piracicaba " [...].

Le " mal de Sigatoka" a été signalé en 1942 dans l'Amazonie et identifié à São Paulo en 1952 [...].

Pour les marchés sud-américains (Argentine, Uruguay), les régimes sont arrimés dans les cales ventilées ou sur le pont des bateaux dont les voyages durent de deux jours et demi à trois jours. Pour les pays d'Europe dont le voyage dure de 15 à 17 jours (Angleterre, Allemagne et autres pays), les régimes devront être emballés et sont embarqués dans des chambres frigorifiques.

A Santos, il existe des fabriques de farine de banane, de pâte de banane et d'autres produits alimentaires. "

....et de l'article de Pierre Subra "Caractères de la culture bananière en Jamaïque " :

"Le facteur essentiel de l'évolution réside dans le changement de variété à la suite de la destruction progressive du Gros-Michel par la maladie de Panama. Cette disparition est de plus en plus complète et il semble que les régions encore peu touchées il y a deux ans [...] le soient maintenant [...]. Il est donc possible de dire que la variété de Jamaïque est le Lacatan [...]. Bien que cette variété soit toujours considérée comme provisoire, il est probable que tant les producteurs que les consommateurs finiront par s'y habituer.

Apparue en Jamä̈que vers 1936, la maladie de Sigatoka a fait l'objet de recherches nombreuses, particulièrement du Dr Leach actuellement Directeur de la recherche au Banana Board [...].

Les travaux d'hybridation du bananier ont été entrepris à Trinidad bien avant la dernière guerre [...]. Actuellement il existe deux stades complémentaires :

- l'Imperial College de Trinidad avec le Dr Simmonds étudie, sélectionne et multiplie les parents mâles,

- la station d'hybridation de Bodles (Jamaïque) reçoit les parents mâles sélectionnés, procède aux pollinisations et étudie les hybrides obtenus. "

Dr. Jacky Ganry

Président de la section Fruits tropicaux et subtropicaux 